\title{
The Very Useful Linear Indefinite Equations in Applied Sciences
}

\author{
Lijiang Zeng \\ (Research Centre of Zunyi Normal College, \\ Zunyi 563099, GuiZhou, P. R. China)
}

\begin{abstract}
The number theory is an important branch of mathematics. The paper introduced some methods of solving linear indefinite equation in convergent and the so-called extended Euclid's algorithm, and got two results about linear indefinite equation. And it is very important for our understanding and using linear indefinite equation in many applied sciences.
\end{abstract}

Key words-great common divisor; indefinite equation; convergents; integer lattice-points; xy-plane

\section{INTRODUCTION}

One of an important branch of mathematics is number theory ${ }^{[1-6]}$, in the number theory, linear indefinite equation ${ }^{[7-10]}$ plays a foundation, and its important role. Linear indefinite equation is an irreplaceable tool in many applied sciences, for example, the linear programming in operational research ${ }^{[11-15]}$, have a special part is integer programming, however, the important role of integer programming is linear indeterminate equation.

\section{SOME NOTES ON LINEAR INDEFINITE EQUATION}

In the number theory, the algebraic equation.

Author introduction: Lijiang Zeng (1962 -), male, born in Guizhou Province of China, Professor of Zunyi Normal College, major research field: mathematics and applied mathematics, research direction: algebra and its application, number theory and its application. Have existed search results: ISTP 2 articles, ISSHP 5 articles, Email: ZLJ4383@sina.com. with two variables

$$
a x+b y=c
$$

is called a linear indefinite equation, and it is also called linear Diophantine equation ${ }^{[16-17]}$ sometimes, for which we wish to find integer solutions in $x$ and $y$.

A linear indefinite equation is a type of algebraic equation with two linear variables. For this reason, it is sometimes also called a bilinear Diophantine equation. In this type of equation $a x+b y=c$, we are only interested in the integer solutions in $x$ and $y$.

In this paper we always note the fact "can not exactly divide" in symbol " $\nmid$ ”, and the fact "can exactly divide” in symbol “|”. For example, $a$ can not exactly divide $b$, we note as $a \nmid b$. $a$ can exactly divide $b$, we note as $a \mid b$, and we always note the great common divisor of $a$ and $b$ in symbol “ $\operatorname{gcd}(a, b)$ ”, other letters is similar meaning. We first explained it here, because we will use these symbols repeatedly.

\section{TWO PROPOSITIONS}

Proposition 1. Let $a, b, c$ be integers with not both $a$ and $b$ equal to 0 , and let $d=\operatorname{gcd}(a, b)$. If $d$ can not exactly divide $c$, then the linear indefinite equation

$$
a x+b y=c
$$

has no integer solution. The equation has an integer solution in $x$ and $y$ if and only if $d{ }^{\nmid} c$. Moreover, if $\left(x_{0}, y_{0}\right)$ is a solution of the equation, then the general solution of the 
equation is

$$
(x, y)=\left(x_{0}+\frac{b}{d} \bullet t, y_{0}-\frac{a}{d} \bullet t\right) t \in Z
$$

Proof. Assume that $x$ and $y$ are integers such that $a x+b y=c$. Since $d \mid a$ and $d|b, d| c$. Hence, if $d \nmid c$, there is no integer solutions of the equation.

Now suppose $d \mid c$. There is an integer $k$ such that $c=k d$. Since $d$ is a sum of multiples of $a$ and $b$, we may write $a m+b n=d$.

Multiplying this equation by $k$, we get $a(m k)+b(n c)=d k=c$, so, that $x=m k$ and $y=n k$ is a solution.

For the "only if" part, suppose $x_{0}$ and $y_{0}$ is a solution of the equation. Then $a x_{0}+b y_{0}=c$. Since $d \mid a$ and $d \mid b$, then $d \mid c$.

Observe that the proof of Proposition 1, together with Euclid's algorithm ${ }^{[16]}$, provides us with a practical method to obtain one solution of the equation. In what follows, however, we shall show how to find $z$ and $y$ by using the continued fraction method.

Suppose that $a$ and $b$ are two integers whose gcd is $d$ and we wish to solve indefinite equations

$$
a x-b y=d
$$

We expand $\frac{a}{b}$ as a finite continued fraction with convergents

$$
\left[\frac{P_{0}}{Q_{0}}, \frac{P_{1}}{Q_{1}}, \cdots, \frac{P_{n-1}}{Q_{n-1}}, \frac{P_{n}}{Q_{n}}\right]=\frac{a}{b}
$$

Since $d=\operatorname{gcd}(a, b)$, we must have $a=d a^{\prime}, b=d b^{\prime}$ and $\operatorname{gcd}\left(a^{\prime}, b^{\prime}\right)=1$. Then $\frac{P_{n}}{Q_{n}}=\frac{a^{\prime}}{b^{\prime}}$ and both fractions are in their lowest terms, giving $P_{n}=a^{\prime}, Q_{n}=b^{\prime}$. So equation (3) gives

$$
P_{n} Q_{n-1}-Q_{n} P_{n-1}=a^{\prime} Q_{n-1}-b^{\prime} P_{n-1}=(-1)^{n-1}
$$

Hence

$$
a Q_{n-1}-b P_{n-1}=d a^{\prime} Q_{n-1}-d b^{\prime} P_{n-1}=(-1)^{n-1} d
$$

or

$$
(-1)^{n-1} a Q_{n-1}-(-1)^{n-1} b P_{n-1}=d
$$

A solution to the equation $a x-b y=d$ is therefore given by

$$
\left\{\begin{array}{l}
x=(-1)^{n-1} Q_{n-1} \\
y=(-1)^{n-1} P_{n-1}
\end{array}\right.
$$

To conclude the above analysis, we have the following Proposition for solving the linear indefinite equation $a x-b y=d$ :

Proposition 2. Let the convergents of the finite continued fraction of $\frac{a}{b}$ be as follows:

$$
\left[\frac{P_{0}}{Q_{0}}, \frac{P_{1}}{Q_{1}}, \cdots, \frac{P_{n-1}}{Q_{n-1}}, \frac{P_{n}}{Q_{n}}\right]=\frac{a}{b}
$$

Then the integer solution ${ }^{[18-19]}$ in $x$ and $y$ of the equation $a x-b y=d$ is

$$
\left.\begin{array}{l}
x=(-1)^{n-1} Q_{n-1} \\
y=(-1)^{n-1} P_{n-1}
\end{array}\right\}
$$

To imitate the proof of the above process, the proof of this Proposition 2 can be obtained, and also you can refer to [16].

\section{REMARK AND EXAMPLE}

Remark 1. We have already known a way of solving equations like (3) by applying Euclid's algorithm to $a$ and $b$ and working backwards through the resulting equations (the so-called extended Euclid's algorithm). Our new method here turns out to be equivalent to this since the continued fraction for $\frac{a}{b}$ is derived from Euclid's algorithm However, it is quicker to generate the convergents $\frac{P_{i}}{Q_{i}}$ using the 
recurrence relations than to work backwards through the equations in Euclid's algorithm

$$
\left[1,2, \frac{3}{2}, \frac{5}{3}, \frac{8}{5}, \frac{85}{53}, \frac{93}{58}, \frac{364}{227}\right]
$$

We have $x=(-1)^{n-1} q_{n-1}=(-1)^{7-1} 58=58$

and $y=(-1)^{n-1} p_{n-1}=(-1)^{7-1} 93=93$. That is $364 \cdot 58-227 \cdot 93=1$

Example 1. Use the continued fraction method to solve the following linear indefinite equation: $\mathbf{2 0 7 1 9 x}+\mathbf{1 3 8 7 1} y=\mathbf{1}$. Note first that

$$
\begin{aligned}
& 20719 x+13871 y=1 \Leftrightarrow \\
& 20719 x-(-13871 y)=1
\end{aligned}
$$

Now since $\frac{20719}{13871}$ can be expanded as a finite continued fraction with convergents

$$
\left[\begin{array}{lllll}
1, \frac{3}{2} & \frac{118}{79}, & \frac{829}{555} & \frac{947}{634} & \frac{1776}{1189} \\
\frac{2723}{1823}, \frac{4499}{3012} & \frac{20719}{13871}
\end{array}\right]
$$

We have $x=(-1)^{n-1} q_{n-1}=(-1)^{8-1} 3012=-3012$, and $y=(-1)^{n-1} p_{n-1}=(-1)^{8-1} 4499=-4499$

That is, $20719 \bullet(-3012)-13871 \bullet(-4499)=1$

The linear indefinite equation $a x+b y=d$ can also be interpreted geometrically. If we allow $(x, y)$ to be any real values, then the graph of this equation is a straight line $L$ in the $x y$-plane. The points $(x, y)$ in the plane with integer coordinates $(x, y)$ are the integer lattice-points. Pairs of integers $(x, y)$ satisfying the equation correspond to integer lattice-points $(x, y)$ on $L$. Thus, Proposition 1 tells us that $L$ passes through such a lattice-point if and only if $\operatorname{gcd}(a, b) \mid d$, in which case it passes through infinitely many of them.

Remark 2. In some areas of number theory (see [16]), it may be necessary to solve the following more general form of linear indefinite equation:

$$
a x y+b x+c y=d .
$$

Note first that this type of equation can be reduced to a factorization: multiplying (11) by $a$, adding be to both sides and factoring results in above

$$
(a x+c)(a y+b)=a d+b c .
$$

If $m n$ is a factorization of $a d+b c$ and a divides $n-c$ and $m-b$, an integer solution of (11) is

$$
\left\{\begin{array}{l}
x=\frac{n-c}{a} \\
y=\frac{m-b}{a}
\end{array}\right.
$$

\section{Reference}

[1] Wolfgang M. Schmidt. Simultaneous approximation to algebraic numbers by rationals[J]. Acta Mathematica. 1970 (1).21-26

[2] Fan Y, Liu H, Lluis Puig. Generalized Hamming weights and equivalences of codes[J]. Science in China, Ser. A. 2003(05).32-36

[3] LAN Tianzhu,XU Yancong,WANG Liangbin.

NewExact Solutions of the Generalized Davey-Stewartson and Mikhailov-Shabat Equations[J]. Journal of hangzhou normal university (natural science edition). 2014(06). 23-26

[4] HU. M. Global Solution for Quasilinear Wave Equations with Viscosity and Nonlinear Perturbation[J]. Northeastern Mathematical Journal. 2001(03)

[5] Roland GLOWINSKI, Annalisa QUAINI. On the Numerical Solution to a Nonlinear Wave Equation Associated with the First Painlev着 Equation:an Operator-Splitting Approach[J]. Chinese Annals of Mathematics(Series B). 2013(02).89-96

[6] G.M. Coclite,H. Holden,K.H. Karlsen. Well-posedness of higher-order Camassa-Holm equations[J]. Journal of Differential Equations . 2008 (3). 78-86

[7] Wu W. ON THE CONSTRUCTION OF GROEBNER BASIS OF A POLYNOMIAL IDEAL BASED ON RIQUIER-JANET THEORY[J]. Systems Science and Mathematical Sciences. 1991(03). 43-51 
[8] Oskar Perron. Grundlagen für eine Theorie des Jacobischen Kettenbruchal gorithmus[J]. Mathematische Annalen. 1907 (1).85-89

[9] Zeng L. Equivalence on finitely generated $R[G]$ module[C]. Proceedings of 2011 Asia-Pacific Youth Conference on Communication ( 2011APYCC ) , April,4-6,2011(Hangzhou). 434-436

[10] Zeng L. On the algebraic integers in cyclotomic fields[C], Proceedings of International Conference on Engineering and Business Management(EBM2011), March, 22-24,2011(Wuhan). 2293-2296.32-37

[11] Muneo Chō,Takeaki Yamazaki. An Operator Transform from Class A to the Class of Hyponormal Operators and its Application[J]. Integral Equations and Operator Theory . 2005 (4) . 142-150

[12] Pietro Aiena,Carlos Carpintero,Ennis Rosas. Some characterizations of operators satisfying a-Browder's theorem[J]. Journal of Mathematical Analysis and Applications . 2005 (2). 87-95

[13] Xuefeng Wang. On concentration of positive bound states of nonlinear Schrödinger equations[J]. Communications in Mathematical Physics . 1993 (2) .57-65

[14] Paul H. Rabinowitz. On a class of nonlinear Schrödinger equations[J]. ZAMP Zeitschrift fürangewandte Mathematik und Physik . 1992 (2). 90-95

[15] Y. Xiao,H.Y. Zhang. A note on convergence of semi-implicit Euler methods for stochastic pantograph equations[J]. Computers and Mathematics with Applications . 2010 (4). 120-126

[16] Davenport H. The Higher Arithmetic, 5th edition[M]. Cambridge University Press, London, New York, 1982, 74-76.

[17] Sun Z H. Consecutive Numbers with the Same Legendre Symbol[C]. Proc.Amer. Math. Soc. 2002, 130: 2503-2507.

[18] Mallat S.A theory for multiresolution signal decomposition[C]. IEEE Transactions on Pattern Analysis and Machine Intelligence . 1989, 143-149

[19] Zeng Y, Li Y, Chen D. A HIERARCHY OF INTEGRABLE HAMILTONIAN SYSTEMS WITH NEUMANN TYPE CONSTRAINT[J]. Chinese Annals of Mathematics. 1992(03).64-69 